\title{
Comprehensive Minority Biomedical Program
}

National Cancer Institute

\section{Source}

National Cancer Institute. Comprehensive Minority Biomedical Program. NCI Thesaurus.

Code C19730.

The Comprehensive Minority Biomedical Program (CMBP) represents an effort by the $\mathrm{NCl}$ to broaden participation in cancer-related research and training activities by minorities, individuals with disabilities, and individuals seeking to reenter an active research career after taking time off to attend to family responsibilities. The CMBP also seeks to enhance the effectiveness of programs in cancer treatment and cancer control in reaching the minority community and other historically underserved segments of the general population. 